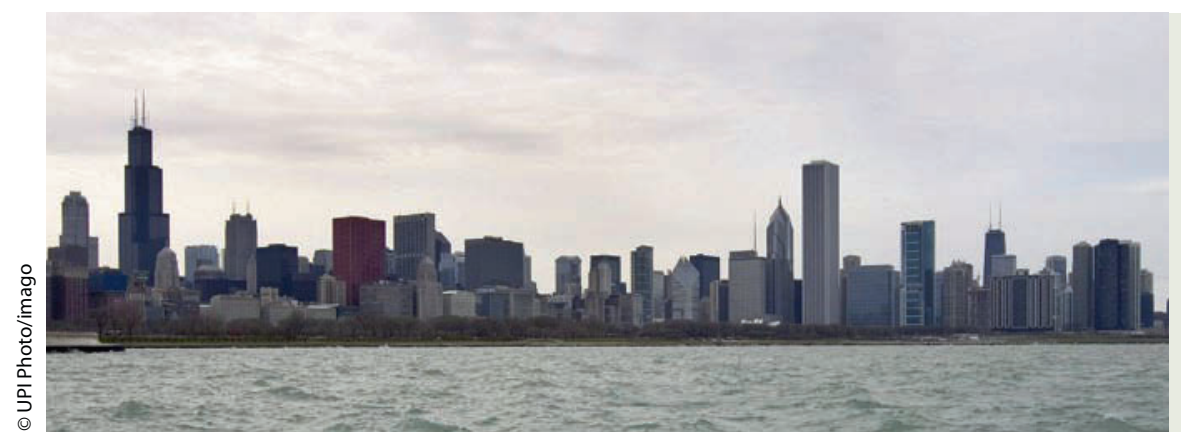

Aktuelle Daten diskutiert

Vom 3.-7. Juni 2011 fand in Chicago das diesjährige Meeting der American Society of Clinical Oncology (ASCO) statt. Aktuellen Studienergebnissen zufolge könnten neue Behandlungsstrategien das Überleben bei fortgeschrittenen Melanomen verlängern.

\title{
ASCO 2011 Annual Meeting
}

\section{Melanomtherapie im Blickpunkt}

\section{ASCQ Neue Therapiestrategien vorgestellt}

Großes Interesse richtet sich auf vielversprechende Behandlungsoptionen - die zielgerichtete molekulare Therapie mit dem BRAF-Inhibitor Vemurafenib und die Immuntherapie mit dem CTL4-Inhibitor Ipilimumab.

Bisher gab es keine Therapie, für die eine Überlebensverlängerung bei Patienten mit metastasiertem Melanom belegt werden konnte. Auf die bisherige Standardtherapie mit dem Alkylans Dacarbazin sprachen nur 15-20\% der Patienten an. Mit hoch dosiertem Interleukin-2 erreichten nur 5-7\% der Patienten eine anhaltende Remission.

Dr. Kim Margolin, Washington, diskutierte neue Therapiestrategien in der Plenarsitzung. Vemurafenib sollte bei Patienten mit aggressivem, symptomatischem Melanom nach positiver Testung auf die aktivierende V600E-Mutation im BRAFGen eingesetzt werden. Interessant war aus ihrer Sicht die Kombination mehrerer Therapiestrategien. So käme eine zusätz- liche Immuntherapie mit Ipilimumab beispielsweise dann zum Einsatz, wenn sich die Tumorlast durch die Vemurafenib-Therapie schon verringert hat. Andererseits könnte bei Patienten mit weniger rasch wachsenden Tumoren Ipilimumab unabhängig vom Mutationsstatus Therapie der ersten Wahl sein und bei Progression Verumafenib folgen.

Die Kombination der beiden Therapiestrategien wird demnächst in einer PhaseI/II-Studie beim metastasiertem Melanom untersucht, ebenso andere Kombinationen wie auch der adjuvante Einsatz.

Andrea Warpakowski

\section{ASC(Immuntherapie mit Überlebensvorteil}

Die Behandlung mit Ipilimumab plus Dacarbazin verbesserte das Gesamtüberleben der Patienten und das Ansprechen des Tumors. Nach drei Jahren lebte noch jeder fünfte Patient. Der rekombinante humanisierte monoklonale Antikörper Ipilimumab hemmt das T-LymphozytenAntigen CTLA-4, das die Aktivierung der Zelle reguliert. Diese Blockade induziert die Produktion von tumorspezifischen T-Lymphozyten.

Als Second-Line-Therapie wurde diese Immuntherapie bereits letztes Jahr auf dem ASCO präsentiert. Nun stellte Prof. Dr. Jedd Wolchok, New York, die Ergebnisse einer doppelblinden Phase-III-Stu- die mit Ipilimumab in der First-LineTherapie vor. Ipilimumab wurde in diesem Fall mit Dacarbacin kombiniert. Insgesamt nahmen 502 nicht vorbehandelte Patienten mit nicht resezierbarem metastasiertem Melanom im Stadium IIIc/IV an der Studie teil. 56\% der Patienten hatten bereits Fernmetastasen (Stadium M1c) und 40\% eine erhöhte Laktatdehydrogenase, die als tumorsekretorischer Marker gilt.

Alle Patienten erhielten zunächst vier Zyklen der folgenden Therapien: Darcarbacin $\left(850 \mathrm{mg} / \mathrm{m}^{2}\right.$ i.v. alle drei Wochen) plus randomisiert entweder Ipilimumab (10 mg/kg i.v. alle drei Wochen) oder Pla- cebo. Diese Induktionsphase wurde mit vier weiteren Zyklen Dacarbazin abgeschlossen. Patienten, die auf die Induktionstherapie ansprachen, wurden wiederum für eine Erhaltungstherapie mit Ipilimumab oder Placebo alle 12 Wochen randomisiert.

\section{Vorteile für Kombination}

Eine komplette Induktionstherapie mit vier Zyklen erhielt nur ein Drittel der Patienten im Ipilimumab/Dacarbazin-Arm gegenüber zwei Drittel im DacarbazinArm. Eine Erhaltungstherapie wurde bei 17,4\% bzw. 21,1\% der Patienten durchgeführt. Trotz dieses Unterschieds lebten nach einem Jahr im Kombinationsarm 47,3\% und im Monotherapiearm 36,3\% der Patienten. Nach drei Jahren lagen die 